\title{
De novo nucleosome assembly: new pieces in an old puzzle
}

\author{
Alain Verreault ${ }^{\mathbf{1}}$ \\ Imperial Cancer Research Fund, Clare Hall Laboratories, South Mimms, EN6 3LD Hertfordshire, UK
}

In eukaryotic cells, DNA packaging within the narrow confines of the cell nucleus is achieved through the formation of chromatin. The basic repeating unit of chromatin is the nucleosome core, which consists of 147 base pairs of DNA wrapped in 1.7 left-handed superhelical turns around the surface of an octameric protein core formed of two molecules each of histones H2A, H2B, H3, and H4. In proliferating cells, the bulk of histone synthesis occurs during $S$ phase of the cell cycle. During DNA replication, the maintenance of chromatin structure is the net result of two fundamentally distinct reactions that take place rapidly during passage of a DNA replication fork. The first reaction, known as parental nucleosome segregation, is the transfer of pre-existing core histones onto the two nascent chromatids behind the fork (Krude 1999). In contrast, the other half of the nucleosome complement is made from newly synthesized histones in a reaction known as de novo nucleosome assembly. This reaction, which is essential for viability in Saccharomyces cerevisiae (Kim et al. 1988), occurs via a stepwise mechanism in which acetylated histones $\mathrm{H} 3$ and $\mathrm{H} 4$ are deposited first and rapidly joined by $\mathrm{H} 2 \mathrm{~A} / \mathrm{H} 2 \mathrm{~B}$ dimers to complete the nucleosome core (Worcel et al. 1978; Smith and Stillman 1991).

Although nucleosomes can be reconstituted in vitro from purified core histones and DNA by salt gradient dialysis, histones and DNA tend to form insoluble aggregates when mixed directly with each other at physiological ionic strength. To circumvent this problem, eukaryotic cells have evolved an elaborate pathway to achieve regulated formation of nucleosomes containing stoichiometric amounts of histones. The purpose of this review is to describe the proteins involved in de novo nucleosome assembly and their functions in the maintenance of chromosome integrity.

\section{Histone acetylation and nucleosome assembly}

Shortly after their synthesis, histones H3 and H4 associate with each other and are acetylated at a number of lysine residues within their amino-terminal domains. In higher eukaryotes, this acetylation is transient and rapidly removed following packaging of the histones into

${ }^{1}$ E-MAIL A.Verreault@icrf.icnet.uk; FAX 442072693801. chromatin (Jackson et al. 1976). Strains of S. cerevisiae in which the acetylation of both $\mathrm{H} 3$ and $\mathrm{H} 4$ is compromised during passage through $\mathrm{S}$ phase exhibit a progressive loss of viability that is accompanied by a decrease in nucleosome density in vivo, and extracts prepared from these cells are defective in nucleosome assembly in vitro (Table 1; Ling et al. 1996; Ma et al. 1998). This strongly suggests that, although the sites of acetylation in $\mathrm{H} 3$ and $\mathrm{H} 4$ are redundant with each other, acetylation of either histone is important to facilitate some aspect of nucleosome assembly during $S$ phase. These experiments have to be interpreted with caution however, because the acetylation site mutations not only prevent histone acetylation but also perturb the structure of the histone amino-terminal domains.

The acetylation of histones prior to their packaging into chromatin is catalyzed by enzymes known as B-type histone acetyltransferases (B-type HATs), which are distinct from A-type HATs that acetylate chromosomal histones. The only known B-type HAT is Hat1, a widely conserved enzyme that, at least in Xenopus and humans, can acetylate lysine residues 5 and 12 of histone H4 (Verreault et al. 1998; Imhof and Wolffe 1999). Although acetylation of these two residues in newly synthesized $\mathrm{H} 4$ is absolutely conserved in a number of widely divergent species (Sobel et al. 1995), hat1s cells have no obvious mutant phenotype in $S$. cerevisiae (Kleff et al. 1995; Parthun et al. 1996). This is not really surprising because Hat 1 is an H4-specific enzyme that cannot modify lysine 8 in $\mathrm{H} 4$. In yeast, the amino termini of histones $\mathrm{H} 3$ and $\mathrm{H} 4$ are redundant with each other, and lysine 8 of $\mathrm{H} 4$ is sufficient to maintain viability and nucleosome assembly even in the absence of an $\mathrm{H} 3$ amino-terminal domain (Table 1). Thus, the lack of phenotype of hat $1 \Delta$ cells may simply reflect the presence of other HATs that can acetylate newly synthesized H3 or H4. The enzyme(s) responsible for these modifications have not yet been identified. It is also not clear whether the B-type H3 HAT will be conserved. Unlike the evolutionarily conserved and very prominent acetylation of newly synthesized histone $\mathrm{H} 4$ on lysines 5 and 12, acetylation of new $\mathrm{H} 3$ molecules seems to occur at different sites in different species, and has not yet been reported in human cells (Fig. 1A; Sobel et al. 1995; Kuo et al. 1996). At least some of the variation in the sites of $\mathrm{H} 3$ acetylation among different species is likely to reflect the 
Table 1. Phenotypes associated with expression of mutant histones during S-phase progression in S. cerevisiae

\begin{tabular}{|c|c|c|}
\hline Histones expressed during S-phase & Cell viability & Chromatin assembly in vitro \\
\hline No $\mathrm{H} 4$ & Inviable (1st cell cycle arrest in G2) & Not determined \\
\hline $\mathrm{H} 3 \Delta \mathrm{N}$ & Viable & Active \\
\hline $\mathrm{H} 4 \Delta \mathrm{N}$ & Viable & Active \\
\hline $\mathrm{H} 3 \Delta \mathrm{N}+\mathrm{H} 4 \Delta \mathrm{N}$ & Inviable (2nd/3rd cell cycle arrest in G2) & Reduced \\
\hline $\mathrm{H} 3 \Delta \mathrm{N}+\mathrm{H} 4 \mathrm{~K} 5 \mathrm{G}, \mathrm{K} 12 \mathrm{G}$ & Viable & Active \\
\hline $\mathrm{H} 3 \Delta \mathrm{N}+\mathrm{H} 4 \mathrm{~K} 5 \mathrm{G}, \mathrm{K} 8 \mathrm{G}, \mathrm{K} 12 \mathrm{G}$ & Inviable (2nd/3rd cell cycle arrest in G2) & Reduced \\
\hline $\mathrm{H} 3 \Delta \mathrm{N}+\mathrm{H} 4 \mathrm{~K} 5 \mathrm{G}, \mathrm{K} 8 \mathrm{G}, \mathrm{K} 16 \mathrm{G}$ & Viable & Active \\
\hline $\mathrm{H} 3 \Delta \mathrm{N}+\mathrm{H} 4 \mathrm{~K} 8 \mathrm{G}, \mathrm{K} 12 \mathrm{G}, \mathrm{K} 16 \mathrm{G}$ & Viable & Active \\
\hline $\mathrm{H} 3 \Delta \mathrm{N}+\mathrm{H} 4 \mathrm{~K} 5 \mathrm{G}, \mathrm{K} 12 \mathrm{G}, \mathrm{K} 16 \mathrm{G}$ & Viable & Active \\
\hline
\end{tabular}

technical difficulties in detecting acetylation sites that are modified infrequently when newly synthesized histones are analyzed by metabolic labeling and amino acid sequencing.

The nucleosome assembly defects due to mutations that completely abolish acetylation of both histones $\mathrm{H} 3$ and $\mathrm{H} 4$ are not as severe as when synthesis of core histones is blocked (Table 1; Ling et al. 1996). Thus, either the main pathway(s) that operates during $S$ phase is not absolutely dependent on histone acetylation or other acetylation-independent nucleosome assembly pathways may exist. Interestingly, a DNA replicationindependent nucleosome assembly assay that is particularly active with extracts prepared from $\mathrm{G}_{2-}, \mathrm{M}-$, and early $G_{1}$-phase cells, requires histones that are less posttranslationally modified than those present during S-phase (Altheim and Schultz 1999). Replication-independent nucleosome assembly may play an important role during the major chromosome structural transitions that occur during mitosis. This pathway, which is known to be active at least in $\mathrm{G}_{2}$ cells (Kim et al. 1988), may also partially complement nucleosome assembly when the activity of the S-phase pathway is compromised by mutations that prevent acetylation of $\mathrm{H} 3$ and $\mathrm{H} 4$.

\section{Taking histones to replicating DNA}

Chromatin assembly factor 1 (CAF-1) was initially identified as a protein that promoted incorporation of newly synthesized H3/H4 into nucleosomes during SV40 DNA replication in vitro (Smith and Stillman 1989). The ability of CAF-1 to promote nucleosome assembly preferentially onto replicating DNA is likely to be important for targeting newly synthesized histones specifically to sites of DNA replication because histones are designed to package DNA in a relatively non-sequence-specific manner. CAF-1's remarkable ability to promote nucleosome formation onto replicating DNA is, at least in part, attributable to its capacity to recognize DNA that is topologically marked by the presence of the proliferating cell nuclear antigen (PCNA; Fig. 1B; Shibahara and Stillman 1999; Moggs et al. 2000). CAF-1 binds directly to PCNA, a DNA polymerase processivity factor that forms a protein clamp around DNA (Fig. 1B). This clamp serves to tether the polymerase to the DNA template, thereby en- suring the processivity of DNA synthesis. In addition to CAF-1, a large number of proteins interact with PCNA during DNA replication (Jonsson and Hubscher 1997). Currently, it is not clear how recruitment of CAF-1, via its interaction with PCNA, is coordinated with that of other DNA replication factors to ensure that newly synthesized histones are specifically deposited onto doublestranded DNA behind the fork without interfering with DNA synthesis.

An attractive possibility for the role of histone acetylation in nucleosome assembly is that chromatin assembly factors need to recognize the acetylation of $\mathrm{H} 3 / \mathrm{H} 4$ to bind to newly synthesized histones. In human cells, CAF-1 exists as a stable complex with newly synthesized $\mathrm{H} 3 / \mathrm{H} 4$ (Fig. 1B). A fraction of the H4 molecules associated with CAF-1 are acetylated on lysines 5, 8, and 12 (Verreault et al. 1996). Intriguingly, any of those three H4 residues needs to be intact to maintain nucleosome assembly and viability in $S$. cerevisiae strains where the amino-terminal domain of $\mathrm{H} 3$ has been completely deleted and therefore cannot be acetylated (Table 1). These observations have led to the widespread view that CAF-1 preferentially binds to acetylated $\mathrm{H} 3 / \mathrm{H} 4$ (Roth and Allis 1996). This is clearly not the case however, because CAF-1 binds stably to $\mathrm{H}_{2} / \mathrm{H}_{2}$ tetramers lacking the amino-terminal domains of both $\mathrm{H} 3$ and $\mathrm{H} 4$ and can promote their efficient assembly during SV40 DNA replication in vitro (Shibahara et al. 2000). Thus, the acetylation of newly synthesized $\mathrm{H} 3 / \mathrm{H} 4$ is likely to facilitate some other aspect of assembly, such as $\mathrm{H} 3 / \mathrm{H} 4$ nuclear import, binding to chromatin assembly factors other than CAF-1 (see RCAF section below), or chromatin maturation.

In human and mouse cells, CAF-1 is present at a large number of DNA replication foci. This is the case both during euchromatin replication early in S-phase and heterochromatin replication later on during S phase (Krude 1995; Taddei et al. 1999), giving the impression that CAF-1-mediated nucleosome assembly is not restricted to particular loci. Paradoxically, CAF-1 is completely dispensable for viability in S. cerevisiae (Enomoto et al. 1997; Kaufman et al. 1997). CAF-1 mutations impair transcriptional silencing of reporter genes integrated next to telomeres and, to a lesser extent, silencing of the mating type loci $H M L \alpha$ and $H M R \mathbf{a}$ (Enomoto et al. 1997; Kaufman et al. 1997; Monson et al. 1997; Enomoto and Berman 1998). At the $H M L \alpha$ locus, CAF-1 is required for 


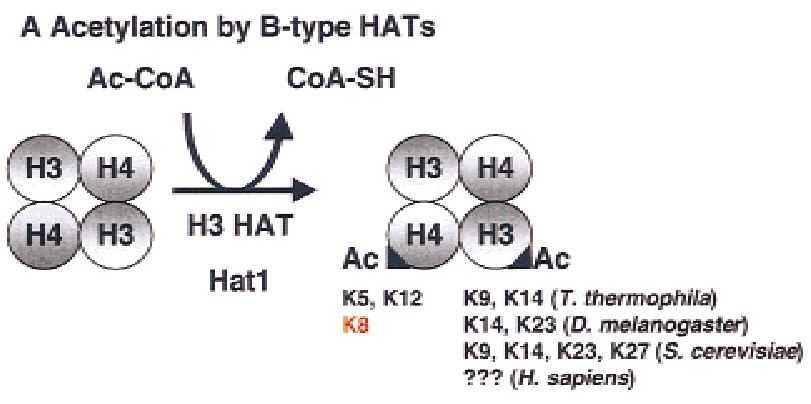

B Targeting to DNA replication fork
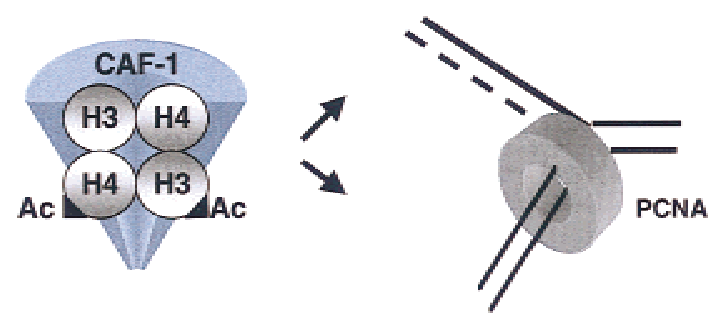

C CAF-1 release and histone deacetylation

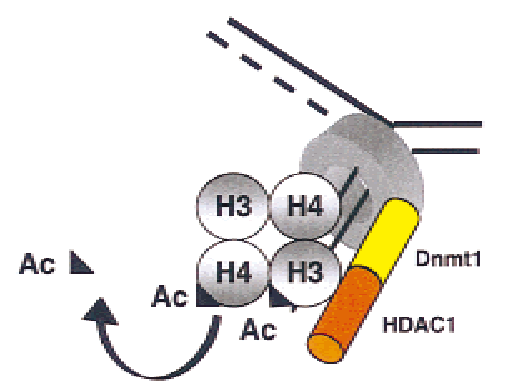

Figure 1. A model for the biochemical steps involved in de novo nucleosome assembly of histones $\mathrm{H} 3 / \mathrm{H} 4$ during DNA replication. $(A)$ The amino termini of $\mathrm{H} 3$ and $\mathrm{H} 4$ are acetylated by at least two distinct B-type histone acetyl transferase (HAT) enzymes. It is not clear whether acetylation occurs before or after $\mathrm{H} 3 / \mathrm{H} 4$ oligomerization. The lysine residues acetylated in newly synthesized $\mathrm{H} 3 / \mathrm{H} 4$ mapped by metabolic labeling and amino acid sequencing are shown in black. Acetylation of lysine 8 in H4 is shown in orange to emphasize that, unlike lysine 5 and 12 acetylation, this modification was not found in metabolic labeling experiments, but was detected in a complex of CAF-1 and H3/H4 isolated from human cells. (B) The CAF-1 protein forms a stable complex with newly synthesized and acetylated $\mathrm{H} 3 / \mathrm{H} 4$ and targets these histones to the DNA replication fork via PCNA. CAF-1 can promote deposition of $\mathrm{H} 3 / \mathrm{H} 4$ onto either the leading (continuous) or the lagging strand (dashed line). (C) Following their deposition onto DNA, enzymes containing HDAC1 may deacetylate newly synthesized $\mathrm{H} 3 / \mathrm{H} 4$. Although this has not been formally established, HDAC1 may be recruited to nascent DNA by virtue of its interaction with the maintenance DNA methyltransferase Dnmt1, an enzyme known to bind directly to PCNA at the DNA replication fork. Clearly, other mechanisms to target histone deacetylases to DNA replication forks probably exist in organisms where DNA methylation is not present.

the maintenance, but not the establishment of transcriptional silencing (Enomoto and Berman 1998). Taken to- gether, these studies suggest that, although dispensable for viability, CAF-1 plays a nonredundant role in the maintenance of heterochromatin at telomeres and the silent mating type loci. Importantly, reporter genes integrated next to telomeres remain packaged into nucleosomes in the absence of CAF-1, and no obvious difference in histone $\mathrm{H} 4$ acetylation of telomere-proximal genes has been found between wild-type and CAF-1 mutant cells (Monson et al. 1997). However, Rap1, a protein involved in both telomeric and mating type silencing, is mislocalized in CAF-1 mutants. In wild-type cells, a major fraction of Rap1 is associated with clusters of telomeres that are often located in the periphery of the cell nucleus. In CAF-1 mutants, telomeric DNA clustering at the periphery of the nucleus is maintained, but the Rap1 foci are more numerous and more diffuse throughout the nucleus (Enomoto et al. 1997). The exact source of the telomeric silencing and Rap1 localization defects seen in the absence of CAF-1 is not clear, but it seems plausible that it may be a result of a subtle change in subtelomeric nucleosomes (e.g., nucleosome spacing and/or a subtle increase in site-specific histone acetylation). Alternatively, the absence of CAF-1 may simply result in a delay in nucleosome assembly during replication that somehow leads to a decrease in the stability of telomeric heterochromatin and dispersion of some of the Rap1 protein throughout the nucleus.

\section{Sensing the need for nucleosome assembly}

In S. cerevisiae, Hir proteins (Hir1, 2, and 3) contribute to histone gene regulation during normal cell cycle progression by repressing transcription of three of the four histone gene pairs, except in late $G_{1}$ and early $S$ phase when the demand for histone synthesis is maximal (Osley and Lycan 1987). In addition, Hir proteins are necessary to repress transcription from the HTA1-HTB1 locus (one of the two gene pairs encoding histones $\mathrm{H} 2 \mathrm{~A}-\mathrm{H} 2 \mathrm{~B}$ ) in response to inhibition of DNA replication or the presence of multiple copies of the HTA and HTB genes (Osley and Lycan 1987; Moran et al. 1990; Sherwood et al. 1993). It is not clear how the Hir proteins can "sense" the need to down-regulate histone gene transcription under these various conditions. A gene encoding a potential mammalian ortholog of the Hir proteins, known as HIRA, may provide a clue to this puzzle. The human HIRA gene is located in a region of chromosome $22 \mathrm{q} 11.2$ that is often deleted in patients with DiGeorge syndrome, a rather frequent haploinsufficiency condition that results in a number of birth defects, including craniofacial, cardiac, and thymic abnormalities (Lamour et al. 1995; Wilming et al. 1997). Interestingly, both the Hira protein and the p60 subunit of CAF-1 bind directly to core histones (Lorain et al. 1998; Shibahara et al. 2000). The role of Hira in mammalian histone gene regulation has not yet been addressed but Hira, the yeast Hir proteins 1 and 2, and the second subunit of CAF-1 (known as p60 in higher eukaryotes or Cac2 in S. cerevisiae), form a subfamily of WD-repeat proteins that are highly related to each other (Lamour et al. 1995; Wilming et al. 1997; 
Kaufman et al. 1998). A very large number of functionally unrelated proteins contain WD-repeat motifs, but the WD repeats of Hira, Hir1, Hir2, p60, and Cac2 are more similar to each other than they are to those of other proteins containing these repeats.

Although HIR gene mutations have relatively mild effects on their own, they exacerbate the transcriptional silencing defects of CAF-1 mutants, and even result in elevated rates of Ty1 retrotransposition, increased sensitivity to the DNA alkylating agent methyl methane sulfonate (MMS) and growth defects (Kaufman et al. 1998; Qian et al. 1998). The MMS sensitivity and growth defects are consistent with a global role for CAF-1 and Hir proteins in promoting nucleosome assembly throughout the genome. This is because DNA damage by MMS is unlikely to occur selectively in heterochromatin, and mutations of the Sir proteins that essentially eliminate heterochromatin-mediated silencing do not result in growth defects. Given the role of Hir proteins in histone gene repression, the more global phenotypes associated with mutations in both CAF-1 and Hir proteins may result from overproduction or untimely expression of newly synthesized histones in cells that lack CAF-1. Alternatively, Hir proteins may themselves play a direct role in nucleosome assembly that becomes more crucial in the absence of CAF-1. A direct role in nucleosome assembly would place the Hir proteins in an ideal position to sense an accumulation of nucleosome assembly precursors when DNA replication is inhibited. The Hir proteins could then trigger a feedback response, ultimately leading to histone gene repression. These two possibilities are not mutually exclusive. Even in the absence of HIR mutations, many perturbations that either increase or decrease histone gene transcription also enhance the telomere silencing defects of CAF-1 mutants (Kaufman et al. 1998). These genetic studies strongly suggest that cells lacking CAF-1 are more susceptible than wild-type cells to fluctuations in the level, the timing of expression or the stoichiometry of newly synthesized histones.

\section{Nucleosome assembly and chromosome repair}

In higher eukaryotic cells, DNA damage leads to perturbations in chromatin structure (Moggs and Almouzni 1999|. De novo nucleosome assembly may therefore play an important role in restoration of chromatin structure following DNA repair. CAF-1 mutants are sensitive to $\mathrm{UV}$, but not ionizing radiation (Kaufman et al. 1997). It is important to emphasize that this UV-sensitive phenotype is relatively mild. CAF-1 mutants are even less UV sensitive than mutants in minor pathways for the repair of UV lesions, such as recombination-mediated repair (Game and Kaufman 1999). The mild UV sensitivity of yeast CAF-1 mutants may be somewhat misleading regarding the potential importance of nucleosome reassembly following DNA repair. This is because CAF-1 is clearly dispensable for nucleosome assembly in vivo. In principle, the mild UV sensitivity of CAF-1 mutants could arise from a subtle defect in chromatin structure that exposes more DNA to UV-induced damage. However, a number of lines of evidence strongly suggest that CAF-1 plays a more direct role in the maintenance of chromosome integrity following UV irradiation.

In human cells, the CAF-1 protein is diffuse throughout the nucleus outside of $S$ phase. However, following UV irradiation of $\mathrm{G}_{1}$ or $\mathrm{G}_{2}$ cells, CAF-1 is rapidly phosphorylated and recruited to foci containing PCNA (Martini et al. 1998). Similar to its role during DNA replication, PCNA is required for the DNA synthesis step of nucleotide excision repair (NER), a major pathway for the repair of UV and other bulky DNA lesions (Lindahl and Wood 1999). Therefore, a significant fraction of the foci containing CAF-1 and PCNA that are formed in response to UV irradiation of $\mathrm{G}_{1}$ - or $\mathrm{G}_{2}$-phase cells are likely to correspond to sites of NER. Consistent with this, CAF-1 can promote nucleosome assembly during DNA repair synthesis in cell-free systems for NER and single-strand break repair (Gaillard et al. 1996; Moggs et al. 2000). Remarkably, histone deposition by CAF-1 during NER does not in any way interfere with the repair process itself. As is the case for nucleosome assembly during DNA replication, histone deposition onto DNA during repair is dependent on CAF-1's ability to interact with PCNA (Moggs et al. 2000). Although this has not yet been strictly demonstrated in vivo, these studies strongly suggest that the role of CAF-1 during NER is to re-establish nucleosome structure. However, this may not be sufficient to account for the UV sensitivity of yeast CAF-1 mutants. This is because DNA synthesis associated with NER only involves the synthesis of short oligonucleotides (Lindahl and Wood 1999). Therefore, the demand for newly synthesized histones to restore nucleosome structure during NER may be relatively modest. Importantly, although CAF-1 mutations enhance the UV sensitivity of both NER and recombination-mediated repair mutants, they do not increase the UV-sensitive phenotype of rad6s or rad18s mutants (Game and Kaufman 1999). Rad6, Rad18, and a number of specialized DNA polymerases are involved in DNA damage tolerance through the bypass of DNA lesions that would otherwise irreversibly block the progression of DNA replication during S phase (Lindahl and Wood 1999). These genetic studies argue that the UV-sensitive phenotype of CAF-1 mutants in yeast is primarily the result of a deficiency in $\operatorname{Rad} 6 / \operatorname{Rad} 18$-dependent DNA lesion bypass mechanisms. Thus, at least in $S$. cerevisiae, the role of CAF-1 in UV damage tolerance may be to promote rapid nucleosome assembly during DNA lesion bypass. Given that both NER (Lindahl and Wood 1999) and Rad6-mediated DNA lesion bypass (Torres-Ramos et al. 1996) require PCNA, it seems likely that CAF-1-dependent nucleosome assembly during DNA repair will be mechanistically similar to nucleosome assembly during normal DNA replication.

RCAF is a novel player in de novo nucleosome assembly that was recently purified from Drosophila embryos based on its ability to synergize with CAF-1 to promote efficient nucleosome assembly during SV40 DNA replication in vitro (Tyler et al. 1999). RCAF is a complex of 
acetylated histones $\mathrm{H} 3$ and $\mathrm{H} 4$, and Asf1, a small, evolutionarily conserved, acidic protein. Importantly, the histones associated with Asf1 as part of RCAF are acetylated at specific lysine residues (lysine 14 of $\mathrm{H} 3$ and lysines 5 and 12 of $\mathrm{H} 4$ ) whose modification is characteristic of newly synthesized H3 and H4 in Drosophila (Fig. 1A; Sobel et al. 1995). Given that acetylation is not necessary for CAF-1 to promote nucleosome assembly (Shibahara et al. 2000), perhaps the binding of Asf1 to histones or its activity in nucleosome assembly are somehow enhanced by site-specific acetylation of either $\mathrm{H} 3$ or H4. In S. cerevisiae, ASF1 was originally identified in two independent screens for genes whose overexpression interfered with telomeric and mating type silencing (Le et al. 1997; Singer et al. 1998). However, like CAF-1 mutants, cells lacking Asf1 are fertile and have no severe silencing defects, although mild silencing defects at telomeres and the mating type loci have been reported (Le et al. 1997; Singer et al. 1998). Unlike CAF-1 mutants, cells lacking Asf1 have an increased doubling time as a result of delayed progression through $\mathrm{G}_{2} / \mathrm{M}$ phase of the cell cycle (Le et al. 1997; Tyler et al. 1999). Cells lacking Asf1 exhibit a number of other phenotypes that are not characteristic of CAF-1 mutants, including a defect in minichromosome maintenance and sensitivity to the DNA replication inhibitor hydroxyurea, the DNA strand break-inducing drug bleomycin and MMS. Some of these phenotypes may be due to a role of Asf1 in promoting nucleosome reassembly during various forms of DNA repair. However, an equally plausible scenario is that the absence of Asf1 simply results in global perturbations in chromatin structure that lead to increased sensitivity to various DNA damaging agents and delayed progression through $\mathrm{G}_{2} / \mathrm{M}$ phase of the cell cycle through activation of DNA damage checkpoints. Consistent with the latter model, defects in chromatin structure due to mutation of multiple lysine residues in the amino-terminal domain of histone $\mathrm{H} 4$ also result in spontaneous damage and a delay in $G_{2} / M$ phase due to activation of the budding yeast Rad9-dependent DNA damage checkpoint (Megee et al. 1995). Cells lacking both Asf1 and CAF-1 are more sensitive to UV radiation and exhibit more pronounced growth and silencing defects than either single mutant. Thus, although both CAF-1 and Asf1 are found associated with newly synthesized and acetylated $\mathrm{H} 3 / \mathrm{H} 4$, these genetic findings argue that the two proteins are functionally distinct and likely to act at different stages of the de novo nucleosome assembly pathway or even in different pathways. Further work is clearly required to establish the precise relationship between Asf1 and CAF-1 in de novo nucleosome assembly during DNA repair and replication.

\section{Deacetylation of newly synthesized histones}

Following their deposition at the DNA replication fork, newly synthesized histones $\mathrm{H} 3 / \mathrm{H} 4$ are rapidly deacetylated (Jackson et al. 1976). At least for some regions of the chromosomes, a number of lines of evidence argue that deacetylation of newly synthesized H3/H4, either during or shortly after DNA replication, is functionally important. In a number of eukaryotes, the nucleosomes present in pericentric heterochromatin contain histone $\mathrm{H} 4$ that is largely unacetylated. In Schizosaccharomyces pombe, either transient exposure to histone deacetylase inhibitors or loss-of-function mutations in the catalytic subunits of two distinct histone deacetylases (Clr3 and Clr6), result in elevated levels of acetylated histone H4 and other structural alterations in pericentric heterochromatin (Ekwall et al. 1997; Grewal et al. 1998). These chromatin structural defects impair kinetochore function and lead to chromosome mis-segregation during mitosis. In late S-phase human cells, newly synthesized H4 is deposited initially during pericentric heterochromatin replication as acetylated molecules modified on lysines 5 and 12. Detectable acetylation of these two residues persists for some period of time, but the acetylation is removed well before entry into mitosis (Taddei et al. 1999), consistent with the $S$. pombe studies arguing that elevated histone $\mathrm{H} 3 / \mathrm{H} 4$ acetylation in pericentric heterochromatin compromises the fidelity of mitotic chromosome segregation.

In mouse cells, pericentric heterochromatin is both deficient in histone acetylation and, due to the presence of large amounts of satellite DNA, extremely rich in methylated CpG dinucleotides. Although the presence of CpG methylated DNA is not a ubiquitous or essential feature of eukaryotic centromeres (no CpG methylation has been found in $S$. cerevisiae, $S$. pombe, or D. melanogaster), two lines of evidence suggest that, in higher eukaryotes, a high density of methylated CpG dinucleotides may play a role in maintaining a low level of histone acetylation in pericentric heterochromatin. First, two distinct DNA-binding proteins that recognize methylated CpG dinucleotides, MeCP2 and MBD2, are particularly abundant in pericentric heterochromatin in mouse cells (Nan et al. 1996; Hendrich and Bird 1998). Both MeCP2 and MBD2 were found to associate with histone deacetylase enzymes that contain the HDAC1 polypeptide as catalytic subunit (Jones et al. 1998; Nan et al. 1998; Ng et al. 1999). Second, the DNA methyltransferase DNMT1, an enzyme responsible for maintenance of CpG methylation during DNA replication, binds directly to HDAC1 (Fuks et al. 2000). Remarkably, targeting of the DNMT1 methyltransferase to DNA replication foci is also dependent on its ability to bind to PCNA (Fig. 1C; Chuang et al. 1997). Although some of the enzymes that contain HDAC1 have been reported to deacetylate substrates other than histones (MartinezBalbas et al. 2000), it seems plausible that at least one of the functions of the interaction between DNMT1 and HDAC1 is to facilitate deacetylation of newly synthesized histones during heterochromatin replication (Fig. 1C). This mechanism would ensure that the acetylation of newly synthesized $\mathrm{H} 3 / \mathrm{H} 4$ that is important for nucleosome assembly during $S$ phase is efficiently removed from pericentric heterochromatin prior to the onset of mitosis, thereby maintaining a chromatin structure that is optimal for centromere function and faithful chromosome segregation (Fig. 2). 


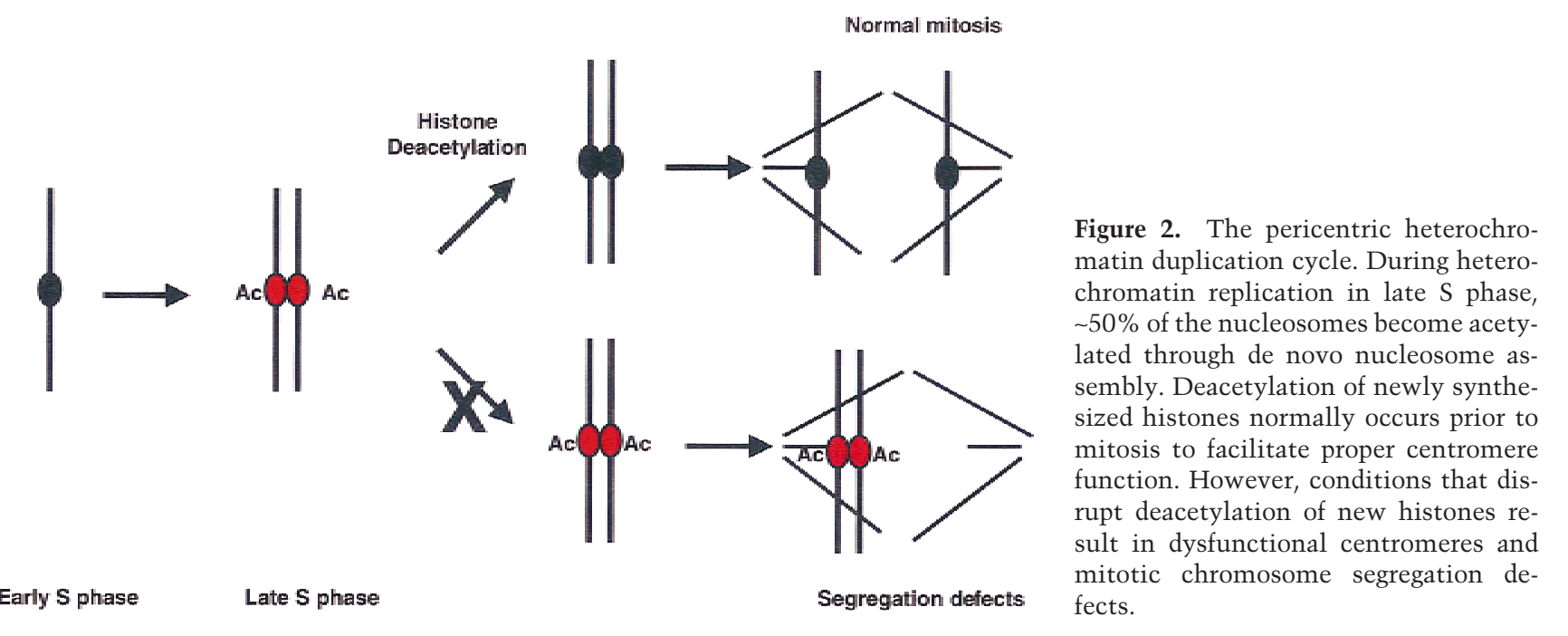

Given that histone acetylation generally tends to promote transcription, deacetylation of newly synthesized histones could also play an important role in transcriptional repression of many genes in proliferating eukaryotic cells. It is not yet clear to what extent this will be important in S. cerevisiae where a large fraction of the genes are expressed and a substantial portion of the histones are acetylated (Davie et al. 1981). This is in striking contrast to higher eukaryotic cells where a large number of genes have to be maintained transcriptionally silent, and only a small fraction of histones are acetylated (Covault and Chalkley 1980; Zhang and Nelson 1988). In S. cerevisiae, at least five genes (RPD3, HDA1, HOS1, HOS2, and HOS3) encode catalytic subunits of histone deacetylases that exhibit sequence similarity with HDAC1. Consistent with a potential role in deacetylation of newly synthesized histones, disruption of $R P D 3$ results in a striking accumulation of chromosomal histone $\mathrm{H} 4$ acetylated at lysines 5 and 12 (Rundlett et al. 1996), the two residues whose acetylation is most characteristic of newly synthesized $\mathrm{H} 4$. However, it is not yet clear whether Rpd3, or any of the other yeast HDAC1-related enzymes, is directly involved in deacetylation of newly synthesized histones.

\section{Beyond the $\mathrm{H} 3 / \mathrm{H} 4$ tetramer: $\mathrm{H} 2 \mathrm{~A} / \mathrm{H} 2 \mathrm{~B}$ assembly and nucleosome spacing}

As is the case for $\mathrm{H}_{2} / \mathrm{H}_{2}$ tetramers, deposition of $\mathrm{H} 2 \mathrm{~A}$ / $\mathrm{H} 2 \mathrm{~B}$ dimers into nucleosomes most likely requires the participation of assembly factors. Nucleoplasmin and $\mathrm{N} 1 / \mathrm{N} 2$ are respectively associated with the large maternal pools of $\mathrm{H} 2 \mathrm{~A} / \mathrm{H} 2 \mathrm{~B}$ and $\mathrm{H} 3 / \mathrm{H} 4$ complexes that are stored in oocytes and utilized for nucleosome assembly during early embryogenesis in Xenopus and Drosophila (Dilworth and Dingwall 1988; Ito et al. 1996b). In these organisms, nucleosome assembly is a particularly active process that is necessary to sustain the rapid rounds of DNA replication and cell division characteristic of early embryogenesis. A general role of nucleoplasmin and N1/
N2 in nucleosome assembly seems unlikely however, because these proteins have only been found in embryonic cells and no homologs have been reported in other eukaryotes. By contrast, a number of lines of evidence suggest that a small acidic protein, known as nucleosome assembly protein 1 (NAP1), may be involved in H2A/H2B assembly. First, NAP1 isolated from a number of different organisms can promote deposition of histone octamers onto DNA at physiological ionic strength (Ito et al. 1997b). This criterion is not by itself sufficient to argue that NAP1 is a genuine assembly factor because other acidic macromolecules such as RNA or polyglutamic acid can also promote histone transfer onto DNA (Ito et al. 1997b). NAP1 is physically associated with newly synthesized histone $\mathrm{H} 2 \mathrm{~A}$ in human cell extracts (Chang et al. 1997) and with a complex of H2A/H2B from Drosophila embryo extracts (Ito et al. 1996a). In addition, both Drosophila NAP1 and human NAP2 (Rodriguez et al. 2000), a protein closely related to NAP1, undergo dramatic changes in their intracellular localization during the course of the cell cycle. NAP2, for instance, is maintained in a phosphorylated and cytoplasmic form outside of $S$ phase. At the $\mathrm{G}_{1}$-to-S-phase transition, NAP2 is dephosphorylated and enters the cell nucleus. Surprisingly, the association of NAP2 with core histones can be detected throughout the cell cycle, but is maximal at the $G_{1} / S$ transition when new histone synthesis is at its peak. In $S$. cerevisiae, there is currently no in vivo evidence supporting a role of Nap1 in nucleosome assembly, although the purified yeast protein has an in vitro nucleosome assembly activity similar to that of its higher eukaryotic homologs (Ishimi and Kikuchi 1991). Paradoxically, biochemical and genetic studies in $S$. cerevisiae and $X$. laevis revealed a role for NAP1 in regulating the functions of protein kinases such as Cdc28-Clb2 and Gin4 during mitosis (Altman and Kellogg 1997 and references therein). Clearly, further work is needed to ascertain the potential role of NAP1 family members in nucleosome assembly in vivo and to determine whether the mitotic functions ascribed to NAP1 in 
yeast and Xenopus are in any way related to its ability to bind to $\mathrm{H} 2 \mathrm{~A} / \mathrm{H} 2 \mathrm{~B}$.

Eukaryotic chromosomes contain long arrays of regularly spaced nucleosome cores that can be revealed by the formation of regular ladders of nuclease-resistant DNA fragments upon treatment with micrococcal nuclease. In contrast, chromatin assembly mediated by either CAF-1 or NAP1 only results in short or very diffuse micrococcal nuclease ladders. In crude chromatin assembly extracts from Drosophila or Xenopus, the appearance of regular nucleosome spacing is an ATP-dependent process (Glikin et al. 1984; Kamakaka et al. 1993). At least three purified multisubunit proteins have been reported to catalyze ATP-dependent nucleosome spacing in vitro. The Drosophila ATP-utilizing chromatin assembly and remodeling factor (ACF) and the chromatin accessibility complex (CHRAC) contain a common subunit known as ISWI (Ito et al. 1997a; VargaWeisz et al. 1997). The human remodeling and spacing factor (RSF) contains a polypeptide highly related to ISWI, known as SNF2h (LeRoy et al. 1998). ISWI serves a catalytic function in ACF and CHRAC by acting as a nucleosome-dependent ATPase. ISWI can promote nucleosome spacing by itself, albeit less efficiently than ACF (Corona et al. 1999; Ito et al. 1999). The potential role of ISWI-containing complexes in nucleosome spacing has not yet been addressed through in vivo experiments. This task is likely to be complicated by the fact that the various protein complexes that contain ISWI can also utilize ATP hydrolysis to disrupt histone-DNA interactions (Tsukiyama and Wu 1995; Ito et al. 1997a; Varga-Weisz et al. 1997). This biochemical property, common to ISWI and many other ATP-dependent chromatin remodeling complexes, has thus far been mostly implicated in transcription (Vignali et al. 2000). Conceivably, these ATP-dependent chromatin remodeling activities could also play a role in various other processes requiring access to genetic information in the context of chromatin.

\section{Concluding remarks}

With the exception of the histones themselves, none of the chromatin assembly factors described in this review are strictly essential for nucleosome assembly or viability in S. cerevisiae. Yet, as argued earlier, histones and DNA do not spontaneously assemble into chromatin under physiological ionic strength conditions in the absence of chromatin assembly factors. These factors are needed to overcome the strong and nonspecific binding of histones to DNA. As a result, proteins such as CAF-1, act both by preventing histones from forming insoluble aggregates with DNA, and by ensuring that newly synthesized histones are selectively targeted to sites of DNA synthesis. The fact that disruption of the genes encoding Hat1, CAF-1, or Asf1 only results in relatively modest phenotypes argues that many other chromatin assembly factors remain to be identified. Although the cast is far from complete, nucleosome assembly during $S$ phase clearly relies on an intricate interplay between chroma- tin assembly factors, histone, and DNA synthesis. As revealed by studies of CAF-1 and Asf1 mutants, perturbations in this delicate balance result in chromosome alterations and susceptibility to DNA damage.

\section{Acknowledgments}

I thank the members of my laboratory for their critical comments and apologize to colleagues whose original references were not included in this manuscript due to space limitations.

\section{References}

Altheim, B.A. and M.C. Schultz. 1999. Histone modification governs the cell cycle regulation of a replication-independent chromatin assembly pathway in Saccharomyces cerevisiae. Proc. Natl. Acad. Sci. 96: 1345-1350.

Altman, R. and D. Kellogg. 1997. Control of mitotic events by Nap1 and the Gin4 kinase. J. Cell. Biol. 138: 119-130.

Chang, L., S.S. Loranger, C. Mizzen, S.G. Ernst, C.D. Allis, and A.T. Annunziato. 1997. Histones in transit: Cytosolic histone complexes and diacetylation of $\mathrm{H} 4$ during nucleosome assembly in human cells. Biochemistry 36: 469-480.

Chuang, L.S., H.I. Ian, T.W. Koh, H.H. Ng, G. Xu, and B.F. Li. 1997. Human DNA-(cytosine-5) methyltransferase-PCNA complex as a target for p21WAF1. Science 277: 1996-2000.

Corona, D.F., G. Langst, C.R. Clapier, E.J. Bonte, S. Ferrari, J.W. Tamkun, and P.B. Becker. 1999. ISWI is an ATP-dependent nucleosome remodeling factor. Mol. Cell 3: 239-245.

Covault, J. and R. Chalkley. 1980. The identification of distinct populations of acetylated histone. J. Biol. Chem. 255: 9110 9116.

Davie, J.R., C.A. Saunders, J.M. Walsh, and S.C. Weber. 1981. Histone modifications in the yeast $S$. cerevisiae. Nucleic Acids Res. 9: 3205-3216.

Dilworth, S.M. and C. Dingwall. 1988. Chromatin assembly in vitro and in vivo. BioEssays 9: 44-49.

Ekwall, K., T. Olsson, B.M. Turner, G. Cranston, and R.C. Allshire. 1997. Transient inhibition of histone deacetylation alters the structural and functional imprint at fission yeast centromeres. Cell 91: 1021-1032.

Enomoto, S. and J. Berman. 1998. Chromatin assembly factor-1 contributes to the maintenance, but not the re-establishment, of silencing at the yeast silent mating loci. Genes \& Dev. 12: 219-232.

Enomoto, S., P.D. McCune-Zierath, M. Gerami-Nejad, M.A. Sanders, and J. Berman. 1997. RLF2, a subunit of yeast chromatin assembly factor-1, is required for telomeric chromatin function in vivo. Genes \& Dev. 11: 358-370.

Fuks, F., W.A. Burgers, A. Brehm, L. Hughes-Davies, and T. Kouzarides. 2000. DNA methyltransferase Dnmt1 associates with histone deacetylase activity. Nat. Genet. 24: 8891.

Gaillard, P.H., E.M. Martini, P.D. Kaufman, B. Stillman, E. Moustacchi, and G. Almouzni. 1996. Chromatin assembly coupled to DNA repair: A new role for chromatin assembly factor 1. Cell 86: 887-896.

Game, J.C. and P.D. Kaufman. 1999. Role of Saccharomyces cerevisiae chromatin assembly factor- 1 in repair of ultraviolet radiation damage in vivo. Genetics 151: 485-497.

Glikin, G.C., I. Ruberti, and A. Worcel. 1984. Chromatin assembly in Xenopus oocytes: In vitro studies. Cell 37: 33-41.

Grewal, S.I., M.J. Bonaduce, and A.J. Klar. 1998. Histone deacetylase homologs regulate epigenetic inheritance of 
transcriptional silencing and chromosome segregation in fission yeast. Genetics 150: 563-576.

Hendrich, B. and A. Bird. 1998. Identification and characterization of a family of mammalian methyl-CpG binding proteins. Mol. Cell. Biol. 18: 6538-6547.

Imhof, A. and A.P. Wolffe. 1999. Purification and properties of the Xenopus Hatl acetyltransferase: Association with the 14-3-3 proteins in the oocyte nucleus. Biochemistry 38: 13085-13093.

Ishimi, Y. and A. Kikuchi. 1991. Identification and molecular cloning of yeast homolog of nucleosome assembly protein 1 which facilitates nucleosome assembly in vitro. I. Biol. Chem. 266: 7025-7029.

Ito, T., M. Bulger, R. Kobayashi, and J.T. Kadonaga. 1996a. Drosophila NAP-1 is a core histone chaperone that functions in ATP-facilitated assembly of regularly spaced nucleosomal arrays. Mol. Cell. Biol. 16: 3112-3124.

Ito, T., J.K. Tyler, M. Bulger, R. Kobayashi, and J.T. Kadonaga. 1996b. ATP-facilitated chromatin assembly with a nucleoplasmin-like protein from Drosophila melanogaster. J. Biol. Chem. 271: 25041-25048.

Ito, T., M. Bulger, M.J. Pazin, R. Kobayashi, and J.T. Kadonaga. 1997a. ACF, an ISWI-containing and ATP-utilizing chromatin assembly and remodeling factor. Cell 90: 145-155.

Ito, T., J.K. Tyler, and J.T. Kadonaga. 1997b. Chromatin assembly factors: A dual function in nucleosome formation and mobilization? Genes Cells 2: 593-600.

Ito, T., M.E. Levenstein, D.V. Fyodorov, A.K. Kutach, R. Kobayashi, and J.T. Kadonaga. 1999. ACF consists of two subunits, Acf1 and ISWI, that function cooperatively in the ATP-dependent catalysis of chromatin assembly. Genes \& Dev. 13: 1529-1539.

Jackson, V., A. Shires, N. Tanphaichitr, and R. Chalkley. 1976. Modifications to histones immediately after synthesis. $I$. Mol. Biol. 104: 471-483.

Jones, P.L., G.J. Veenstra, P.A. Wade, D. Vermaak, S.U. Kass, N. Landsberger, J. Strouboulis, and A.P. Wolffe. 1998. Methylated DNA and MeCP2 recruit histone deacetylase to repress transcription. Nat. Genet. 19: 187-191.

Jonsson, Z.O. and U. Hubscher. 1997. Proliferating cell nuclear antigen: More than a clamp for DNA polymerases. BioEssays 19: 967-975.

Kamakaka, R.T., M. Bulger, and J.T. Kadonaga. 1993. Potentiation of RNA polymerase II transcription by Gal4-VP16 during but not after DNA replication and chromatin assembly. Genes \& Dev. 7: 1779-1795.

Kaufman, P.D., R. Kobayashi, and B. Stillman. 1997. Ultraviolet radiation sensitivity and reduction of telomeric silencing in Saccharomyces cerevisiae cells lacking chromatin assembly factor-1. Genes \& Dev. 11: 345-357.

Kaufman, P.D., J.L. Cohen, and M.A. Osley. 1998. Hir proteins are required for position-dependent gene silencing in Saccharomyces cerevisiae in the absence of chromatin assembly factor 1. Mol. Cell. Biol. 18: 4793-4806.

Kim, U.J., M. Han, P. Kayne, and M. Grunstein. 1988. Effects of histone $\mathrm{H} 4$ depletion on the cell cycle and transcription of Saccharomyces cerevisiae. EMBO J. 7: 2211-2219.

Kleff, S., E.D. Andrulis, C.W. Anderson, and R. Sternglanz. 1995. Identification of a gene encoding a yeast histone $\mathrm{H} 4$ acetyltransferase. J. Biol. Chem. 270: 24674-24677.

Krude, T. 1995. Chromatin assembly factor-1 (CAF-1) colocalizes with replication foci in HeLa cell nuclei. Exp. Cell. Res. 220: 304-311.

-1999. Chromatin assembly during DNA replication in somatic cells. Eur. J. Biochem. 263: 1-5.

Kuo, M.H., J.E. Brownell, R.E. Sobel, T.A. Ranalli, R.G. Cook,
D.G. Edmondson, S.Y. Roth, and C.D. Allis. 1996. Transcription-linked acetylation by Gen5p of histones H3 and H4 at specific lysines. Nature 383: 269-272.

Lamour, V., Y. Lecluse, C. Desmaze, M. Spector, M. Bodescot, A. Aurias, M.A. Osley, and M. Lipinski. 1995. A human homolog of the $S$. cerevisiae HIR1 and HIR2 transcriptional repressors cloned from the DiGeorge syndrome critical region. Hum. Mol. Genet. 4: 791-799.

Le, S., C. Davis, J.B. Konopka, and R. Sternglanz. 1997. Two new S-phase-specific genes from Saccharomyces cerevisiae. Yeast 13: 1029-1042.

LeRoy, G., G. Orphanides, W.S. Lane, and D. Reinberg. 1998. Requirement of RSF and FACT for transcription of chromatin templates in vitro. Science 282: 1900-1904.

Lindahl, T. and R.D. Wood. 1999. Quality control by DNA repair. Science 286: 1897-1905.

Ling, X., T.A. Harkness, M.C. Schultz, G. Fisher-Adams, and M. Grunstein. 1996. Yeast histone H3 and H4 amino termini are important for nucleosome assembly in vivo and in vitro: Redundant and position-independent functions in assembly but not in gene regulation. Genes \& Dev. 10: 686-699.

Lorain, S., J.P. Quivy, F. Monier-Gavelle, C. Scamps, Y. Lecluse, G. Almouzni, and M. Lipinski. 1998. Core histones and HIRIP3, a novel histone-binding protein, directly interact with WD repeat protein HIRA. Mol. Cell. Biol. 18: 5546-5556.

Ma, X.J., J. Wu, B.A. Altheim, M.C. Schultz, and M. Grunstein. 1998. Deposition-related sites K5/K12 in histone H4 are not required for nucleosome deposition in yeast. Proc. Nat1. Acad. Sci. 95: 6693-6698.

Martinez-Balbas, M.A., U.M. Bauer, S.J. Nielsen, A. Brehm, and T. Kouzarides. 2000. Regulation of E2F1 activity by acetylation. $E M B O$ J. 19: 662-671.

Martini, E., D.M. Roche, K. Marheineke, A. Verreault, and G. Almouzni. 1998. Recruitment of phosphorylated chromatin assembly factor 1 to chromatin after UV irradiation of human cells. J. Cell. Biol. 143: 563-575.

Megee, P.C., B.A. Morgan, and M.M. Smith. 1995. Histone H4 and the maintenance of genome integrity. Genes \& Dev. 9: $1716-1727$.

Moggs, J.G. and G. Almouzni. 1999. Chromatin rearrangements during nucleotide excision repair. Biochimie 81: 45-52.

Moggs, J.G., P. Grandi, J.P. Quivy, Z.O. Jonsson, U. Hubscher, P.B. Becker, and G. Almouzni. 2000. A CAF-1-PCNA-mediated chromatin assembly pathway triggered by sensing DNA damage. Mol. Cell. Biol. 20: 1206-1218.

Monson, E.K., D. de Bruin, and V.A. Zakian. 1997. The yeast Cacl protein is required for the stable inheritance of transcriptionally repressed chromatin at telomeres. Proc. Nat1. Acad. Sci. 94: 13081-13086.

Moran, L., D. Norris, and M.A. Osley. 1990. A yeast H2A-H2B promoter can be regulated by changes in histone gene copy number. Genes \& Dev. 4: 752-763.

Nan, X., P. Tate, E. Li, and A. Bird. 1996. DNA methylation specifies chromosomal localization of MeCP2. Mol. Cell. Biol. 16: 414-421.

Nan, X., H.H. Ng, C.A. Johnson, C.D. Laherty, B.M. Turner, R.N. Eisenman, and A. Bird. 1998. Transcriptional repression by the methyl-CpG-binding protein MeCP2 involves a histone deacetylase complex. Nature 393: 386-389.

Ng, H.H., Y. Zhang, B. Hendrich, C.A. Johnson, B.M. Turner, H. Erdjument-Bromage, P. Tempst, D. Reinberg, and A. Bird. 1999. MBD2 is a transcriptional repressor belonging to the MeCP1 histone deacetylase complex. Nat. Genet. 23: 58-61.

Osley, M.A. and D. Lycan. 1987. Trans-acting regulatory mutations that alter transcription of Saccharomyces cerevisiae histone genes. Mol. Cell. Biol. 7: 4204-4210. 
Parthun, M.R., J. Widom, and D.E. Gottschling. 1996. The major cytoplasmic histone acetyltransferase in yeast: Links to chromatin replication and histone metabolism. Cell 87: 85-94.

Qian, Z., H. Huang, J.Y. Hong, C.L. Burck, S.D. Johnston, J. Berman, A. Carol, and S.W. Liebman. 1998. Yeast Tyl retrotransposition is stimulated by a synergistic interaction between mutations in chromatin assembly factor 1 and histone regulatory proteins. Mol. Cell. Biol. 18: 4783-4792.

Rodriguez, P., J. Pelletier, G.B. Price, and M. Zannis-Hadjopoulos. 2000. NAP-2: Histone chaperone function and phosphorylation state through the cell cycle. J. Mol. Biol. 298: 225-238.

Roth, S.Y. and C.D. Allis. 1996. Histone acetylation and chromatin assembly: A single escort, multiple dances? Cell 87: 5-8.

Rundlett, S.E., A.A. Carmen, R. Kobayashi, S. Bavykin, B.M. Turner, and M. Grunstein. 1996. HDA1 and RPD3 are members of distinct yeast histone deacetylase complexes that regulate silencing and transcription. Proc. Natl. Acad. Sci. 93: $14503-14508$.

Sherwood, P.W., S.V. Tsang, and M.A. Osley. 1993. Characterization of HIR1 and HIR2, two genes required for regulation of histone gene transcription in Saccharomyces cerevisiae. Mol. Cell. Biol. 13: 28-38.

Shibahara, K. and B. Stillman. 1999. Replication-dependent marking of DNA by PCNA facilitates CAF-1-coupled inheritance of chromatin. Cell 96: 575-585.

Shibahara, K.S., A. Verreault, and B. Stillman. 2000. The Nterminal domains of histones $\mathrm{H} 3$ and $\mathrm{H} 4$ are not necessary for chromatin assembly factor 1-mediated nucleosome assembly onto replicated DNA in vitro. Proc. Natl. Acad. Sci. (in press)

Singer, M.S., A. Kahana, A.J. Wolf, L.L. Meisinger, S.E. Peterson, C. Goggin, M. Mahowald, and D.E. Gottschling. 1998. Identification of high-copy disruptors of telomeric silencing in Saccharomyces cerevisiae. Genetics 150: 613-632.

Smith, S. and B. Stillman. 1989. Purification and characterization of CAF-1, a human cell factor required for chromatin assembly during DNA replication. Cell 58: 15-25.

- 1991. Stepwise assembly of chromatin during DNA replication in vitro. EMBO J. 10: 971-980.

Sobel, R.E., R.G. Cook, C.A. Perry, A.T. Annunziato, and C.D. Allis. 1995. Conservation of deposition-related acetylation sites in newly synthesized histones $\mathrm{H} 3$ and H4. Proc. Nat1. Acad. Sci. 92: 1237-1241.

Taddei, A., D. Roche, J.B. Sibarita, B.M. Turner, and G. Almouzni. 1999. Duplication and maintenance of heterochromatin domains. J. Cell. Biol. 147: 1153-1166.

Torres-Ramos, C.A., B.L. Yoder, P.M. Burgers, S. Prakash, and L. Prakash. 1996. Requirement of proliferating cell nuclear antigen in RAD6-dependent postreplicational DNA repair. Proc. Natl. Acad. Sci. 93: 9676-9681.

Tsukiyama, T. and C. Wu. 1995. Purification and properties of an ATP-dependent nucleosome remodeling factor. Cell 83: $1011-1020$.

Tyler, J.K., C.R. Adams, S.R. Chen, R. Kobayashi, R.T. Kamakaka, and J.T. Kadonaga. 1999. The RCAF complex mediates chromatin assembly during DNA replication and repair. Nature 402: 555-560.

Varga-Weisz, P.D., M. Wilm, E. Bonte, K. Dumas, M. Mann, and P.B. Becker. 1997. Chromatin-remodelling factor CHRAC contains the ATPases ISWI and topoisomerase II. Nature 388: $598-602$.

Verreault, A., P.D. Kaufman, R. Kobayashi, and B. Stillman. 1996. Nucleosome assembly by a complex of CAF-1 and acetylated histones H3/H4. Cell 87: 95-104.
1998. Nucleosomal DNA regulates the core-histonebinding subunit of the human Hatl acetyltransferase. Curr. Biol. 8: 96-108.

Vignali, M., A.H. Hassan, K.E. Neely, and J.L. Workman. 2000. ATP-dependent chromatin-remodeling complexes. Mol. Cell. Biol. 20: 1899-1910.

Wilming, L.G., C.A. Snoeren, A. van Rijswijk, F. Grosveld, and C. Meijers. 1997. The murine homologue of HIRA, a DiGeorge syndrome candidate gene, is expressed in embryonic structures affected in human CATCH22 patients. Hum. Mol. Genet. 6: 247-258.

Worcel, A., S. Han, and M.L. Wong. 1978. Assembly of newly replicated chromatin. Cell 15: 969-977.

Zhang, D.E. and D.A. Nelson. 1988. Histone acetylation in chicken erythrocytes. Rates of acetylation and evidence that histones in both active and potentially active chromatin are rapidly modified. Biochem. J. 250: 233-240. 


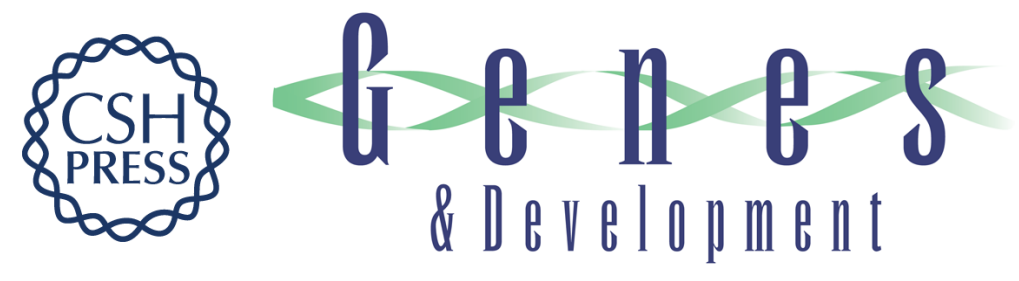

\section{De novo nucleosome assembly: new pieces in an old puzzle}

Alain Verreault

Genes Dev. 2000, 14:

Access the most recent version at doi:10.1101/gad.14.12.1430

References This article cites 75 articles, 39 of which can be accessed free at: http://genesdev.cshlp.org/content/14/12/1430.full.html\#ref-list-1

License

Email Alerting Receive free email alerts when new articles cite this article - sign up in the box at the top Service right corner of the article or click here.

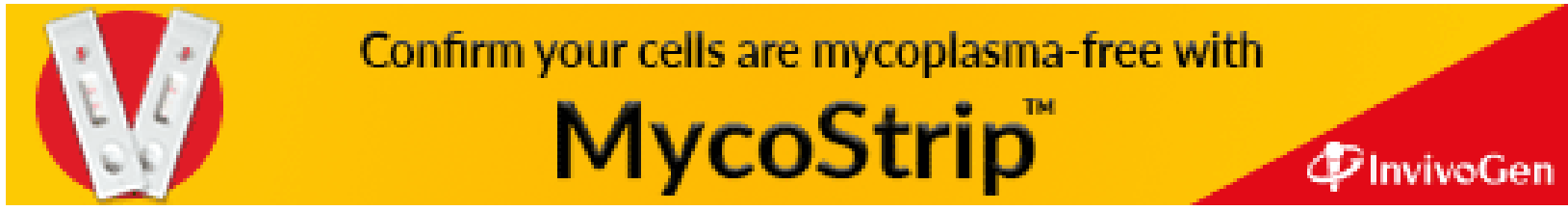

